\title{
Pyrethrin and Pyrethroid Exposures in the United States: A Longitudinal Analysis of Incidents Reported to Poison Centers
}

\author{
Laura E. Power, MSa, Daniel L. Sudakin, MD, MPH, FACMT, FACOEMa
}

aDepartment of Environmental and Molecular Toxicology, Oregon State University, 333 Weniger, Corvallis, OR 97331-6502

\begin{abstract}
Introduction: Citing the Food Quality Protection Act, the US Environmental Protection Agency (EPA) decided to phase out and eliminate organophosphate insecticide use in residential environments. The phase out process spanned from 2000 to 2005 , and it may have resulted in increased consumer use of insecticides containing other active ingredients. This study utilized data from the national Poison Control Center to assess possible changes in exposure incidents involving pyrethrin and pyrethroid insecticides during the phase out of organophosphates from residential uses.

Methods: We extracted pyrethrin and pyrethroid insecticide exposure data from the American Association of Poison Control Centers Toxic Exposure Surveillance System (TESS) annual reports from 2000 to 2005. We examined pyrethrin and pyrethroid incidents by total exposures for each year, and we stratified exposures by age range, reason, number of cases treated in a health care facility, and medical outcome. Cases were examined as a proportion of all insecticide exposures. We calculated the annual incidence rates for exposures involving pyrethrin and pyrethroid insecticides of the population served.

Results: Pyrethrin and pyrethroid exposures increased annually in number and as a percentage of all insecticide exposure incidents. The increase in cases was observed for all age categories and exposure reasons. A statistically significant correlation was observed between advancing years (2000-2005) and the number of pyrethrin and pyrethroid incidents $(\mathrm{p}<.01)$. While the number of incidents treated in a health care facility increased annually during the study period, the proportion was constant $(0.185 \pm 0.012)$ over the sixyear period. During the study period, the incidence rate for pyrethrin and pyrethroid exposures increased each year. $44 \%$ of all medical outcomes data was recorded, and the majority of outcomes resulted in no symptoms (37\%) or minor symptoms (53\%).

Conclusion: TESS data showed a clear increase in cases involving pyrethrins and pyrethroids. The increase was temporally associated with the phase out of organophosphates from residential uses. In the future, medical toxicologists and poison control center personnel should be prepared to respond to an increasing number of pyrethrin and pyrethroid insecticide exposures.
\end{abstract}

\section{INTRODUCTION}

The Food Quality Protection Act (FQPA) of 1996 resulted in a risk mitigation decision by the United States Environmental Protection Agency (US EPA) to phase out and eliminate uses of organophosphate insecticides in residential (home and garden) environments and certain agricultural (food) uses [1,2]. The phase out from residential uses started in the year 2000 and concluded at the end of $2005[3,4]$. Our previous f longitudinal analysis of national Poison Control Center (PCC) data determined there was a significant decline in the number of organophosphate exposures after the commencement of the phase out period [5]. The

Keywords: pyrethrin insecticides, pyrethroid insecticides, pesticide exposures, Poison Control Center data, epidemiology Notes: The authors receive support through U.S. EPA Cooperative Agreement 83239901 (National Pesticide Medical Monitoring Program).

Corresponding Author: Laura E. Power, MS, Department of Environmental and Molecular Toxicology, Oregon State University, 333 Weniger, Corvallis, OR 97331-6502. Email: powerl@science.oregonstate.edu 
results of our longitudinal study were consistent with other investigations that reported on the complicated effect access to pesticides can have on the epidemiology of pesticide exposures, including human poisonings $[6,7]$.

The phase out of organophosphate pesticides from residential uses may have resulted in an increased consumer use of other insecticide formulations containing different types of active ingredients. Pyrethrins and pyrethroids are two distinct classes of insecticides widely available to consumers. Pyrethrin insecticides are extracted from the flowers of Chrysanthemum cinerariaefolium (pyrethrum), and they are esters of chrysanthemic acid and pyrethric acid [8]. Pyrethrins break down quickly in the presence of sunlight, and so more photostable synthetic analogues, called the pyrethroids, were developed as insecticides for the outdoor environment.

Pyrethroid insecticides mainly effect voltage-gated sodium channels. Pyrethroids alter the gating characteristics of neuronal sodium channels, delaying their closure and resulting in a lowered action potential threshold and repetitive firing $[9,10]$. Although the mechanism of action of pyrethrins has not been as thoroughly investigated as pyrethroids, a review concluded that pyrethrins are also considered sodium channel toxins that possess a similar toxicodynamic effect as pyrethroids [11]. Pyrethrins and pyrethroids have a lower degree of mammalian and human toxicity than insects because insects have increased sodium channel sensitivity, a smaller body size, and lower body temperature [9].

As national PCC statistics have demonstrated a significant reduction in organophosphate exposures in association with their phase-out from residential uses, it would be important to assess whether there has been a concurrent change in the number of incidents involving other common insecticides. Given the widespread use and availability to consumers, evaluating pyrethrin and pyrethroid insecticides is important. The purpose of this investigation was to utilize national PCC data to assess whether there has been a change in exposures involving pyrethrin and pyrethroid insecticides during the phase out period of organophosphates from residential uses.

\section{MATERIALS AND METHODS}

We extracted pyrethrin and pyrethroid insecticide exposure data from the TESS annual reports [12-17]. TESS is a uniform data set of exposure incidents reported to PCCs in the United States. Data includes information relating to patient demographics, the circumstances surrounding exposure (unintentional vs. intentional), the substance(s), medical outcome, and other relevant endpoints [18]. Regional PCCs provide data for TESS and to the American Association of Poison Control Centers data. Cases reported as confirmed nonexposure by PCCs are not included in TESS reports and therefore not included in this study [17]. TESS data represented $100 \%$ of human exposures reported to poison centers in the United States during 2003, 2004, and 2005, increasing from 83\% in 1995 and 96\% in 1999 [15-20]. These data are published annually in the scientific literature [16]. The investigation went from 2000-2005 and includes the phase out period and cancellation of organophosphates from residential applications in the United States.

We examined all exposures involving pyrethrin and/or pyrethroid insecticides for each year. Data were stratified by:

1. age range (age $<6$ years, $6-19$ years, $>19$ years),

2. reason for exposure (unintentional and intentional),

3. number of cases treated in a health care facility,

4. and medical outcome (no effects, minor, moderate, major, and death).

TESS Annual Reports do not include data on gender distribution for pesticide exposures, and so gender was not included in the analyses. Annual incidence rates for exposures involving pyrethrin and pyrethroid insecticides for the population served by the PCCs were calculated from 2000 to 2005 . The population size served by PCCs for each year were extracted from the 2005 TESS Annual Report, and it served as the denominator for the calculation of incidence rates.

The proportion of pyrethrin and/or pyrethroid exposures with data documenting the medical outcome was calculated for each year. Medical outcomes were not assigned to all reported exposures for a number of reasons: in some cases the responding poison center determined a scenario was most likely nontoxic or minimally toxic; some cases were not followed and determined potentially toxic; or the exposure was not responsible for the effect [21]. As TESS includes data on other classes of insecticides (such as organophosphates and N-methylcarbamates), we calculated the proportion of all insecticide exposures that involved pyrethrin and pyrethroid formulations for each year. SPSS was used to test the hypothesis that a positive correlation existed between advancing years of the study period and the number of pyrethrin and pyrethroid incidents (Spearman's rho).

\section{RESULTS}

Based upon TESS data collected in the United States, a total of 121,748 pyrethrin and pyrethroid exposures were reported to PCCs from 2000 to 2005. Table 1 and Figure 1 summarize data on pyrethrin and pyrethroid exposures during the study period. Pyrethrin and pyrethroid exposures increased annually in number and as a percentage of all insecticide cases. The number of pyrethrin and pyrethroid exposures increased from 13,759 in 2000 to 25,949 in 2005 . A statistically significant correlation was observed between advancing years (2000-2005) and the number of pyrethrin and pyrethroid incidents (Spearman's rho, $\mathrm{p}<.01$ ). The proportion of all insecticide exposures involving pyrethrins and pyrethroids increased from 30\% in the year 2000 to $47 \%$ in 2005 (Figure 1). The number of incidents that were treated in a health care facility increased from 2,825 in 2000 to 4,934 in 2005, while the proportion of cases treated in a health care facility was constant $(0.185 \pm 0.012)$ during the study period.

The increase in the number of pyrethrin and pyrethroid exposures during the study period from 2000 to 2005 was apparent 
Table 1: Pyrethrin and pyrethroid exposures in the United States (Toxic Exposure Surveillance System Data, 2000-2005)

\begin{tabular}{|c|c|c|c|c|c|c|c|}
\hline \multirow[b]{2}{*}{ Year } & \multirow{2}{*}{$\begin{array}{l}\text { Total No. of } \\
\text { Exposures }\end{array}$} & \multicolumn{3}{|c|}{ Age (Years) } & \multicolumn{2}{|c|}{ Exposure Reason } & \multirow{2}{*}{$\begin{array}{c}\text { Treated in } \\
\text { Health Care } \\
\text { Facility }\end{array}$} \\
\hline & & $<6$ & 6-19 & $>19$ & Unintentional & Intentional & \\
\hline 2000 & 13,759 & 4,926 & 1,735 & 6,945 & 12,859 & 392 & 2,825 \\
\hline 2001 & 17,294 & 5,828 & 2,138 & 9,174 & 16,094 & 427 & 3,158 \\
\hline 2002 & 19,442 & 6,476 & 2,193 & 10,573 & 18,032 & 531 & 3,503 \\
\hline 2003 & 21,135 & 6,787 & 2,353 & 11,661 & 19,607 & 507 & 3,776 \\
\hline 2004 & 24,169 & 7,387 & 2,653 & 13,877 & 22,355 & 646 & 4,126 \\
\hline 2005 & 25,949 & 7,673 & 2,820 & 15,211 & 24,089 & 683 & 4,934 \\
\hline
\end{tabular}

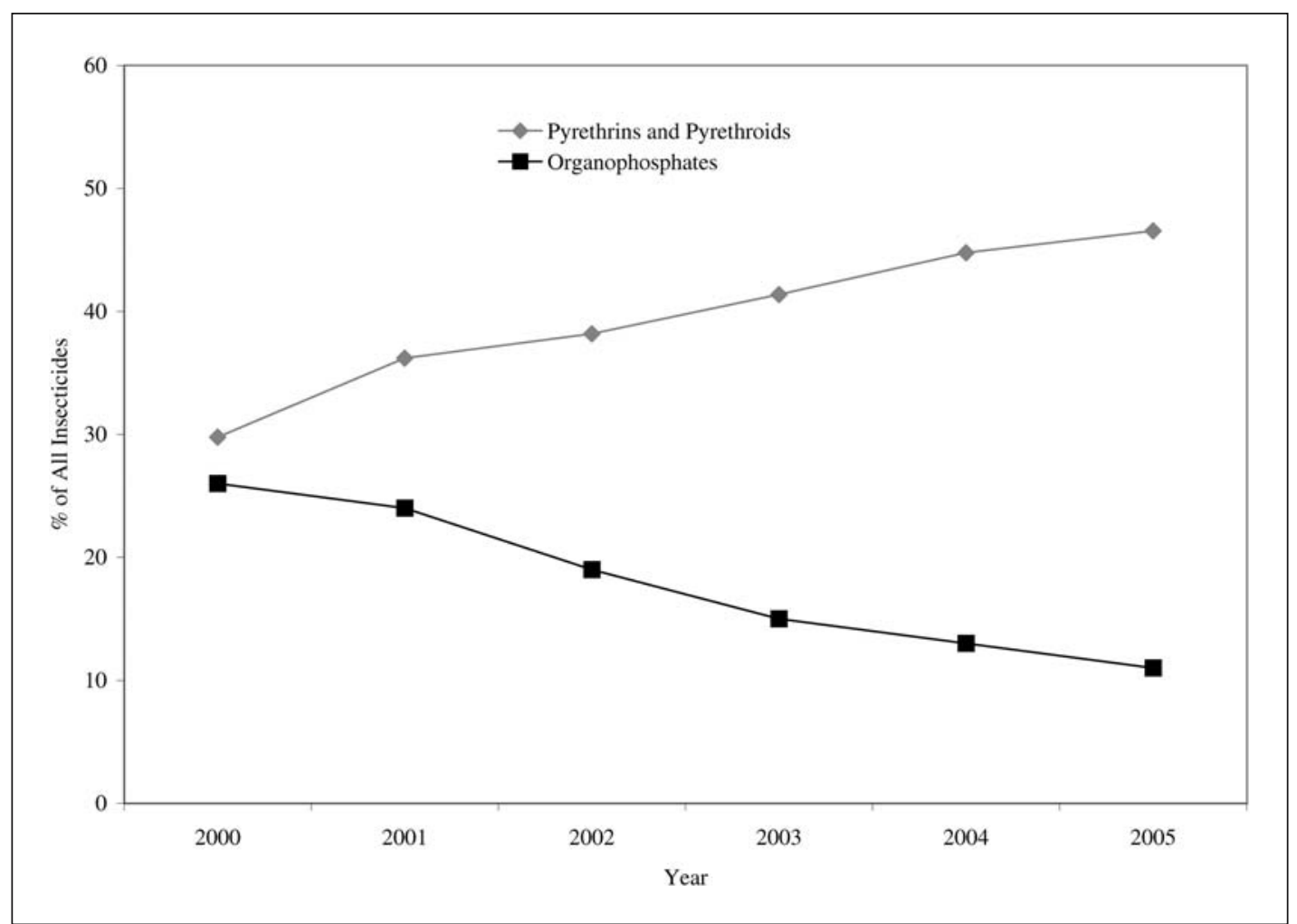

Figure 1: Percent of all insecticide exposure incidents involving pyrethrins and pyrethroids, and organophosphates* by year.

*Organophosphate data taken from [5,17].

across all age categories. Throughout the study period, the majority $(92.9 \pm 0.3 \%)$ of exposures were classified as unintentional (accidental). An increase in the number of unintentional exposures was observed annually from 2000 to 2005, while there was a general pattern of increase in the number of intentional exposures from 2000 to 2005. Figure 2 shows the annual incidence rate of all pyrethrin, pyrethroid, and organophosphate exposures per
100,000 population from 2000 to 2005 . The incidence rate for pyrethrins and pyrethroids increased each year from 5.08 exposures per 100,000 population in 2000 to 8.75 exposures per 100,000 population in 2005.

Table 2 summarizes the medical outcomes of pyrethrin and pyrethroid exposures for the years 2000 to 2005. Medical outcomes were not recorded for the majority of the cases. 


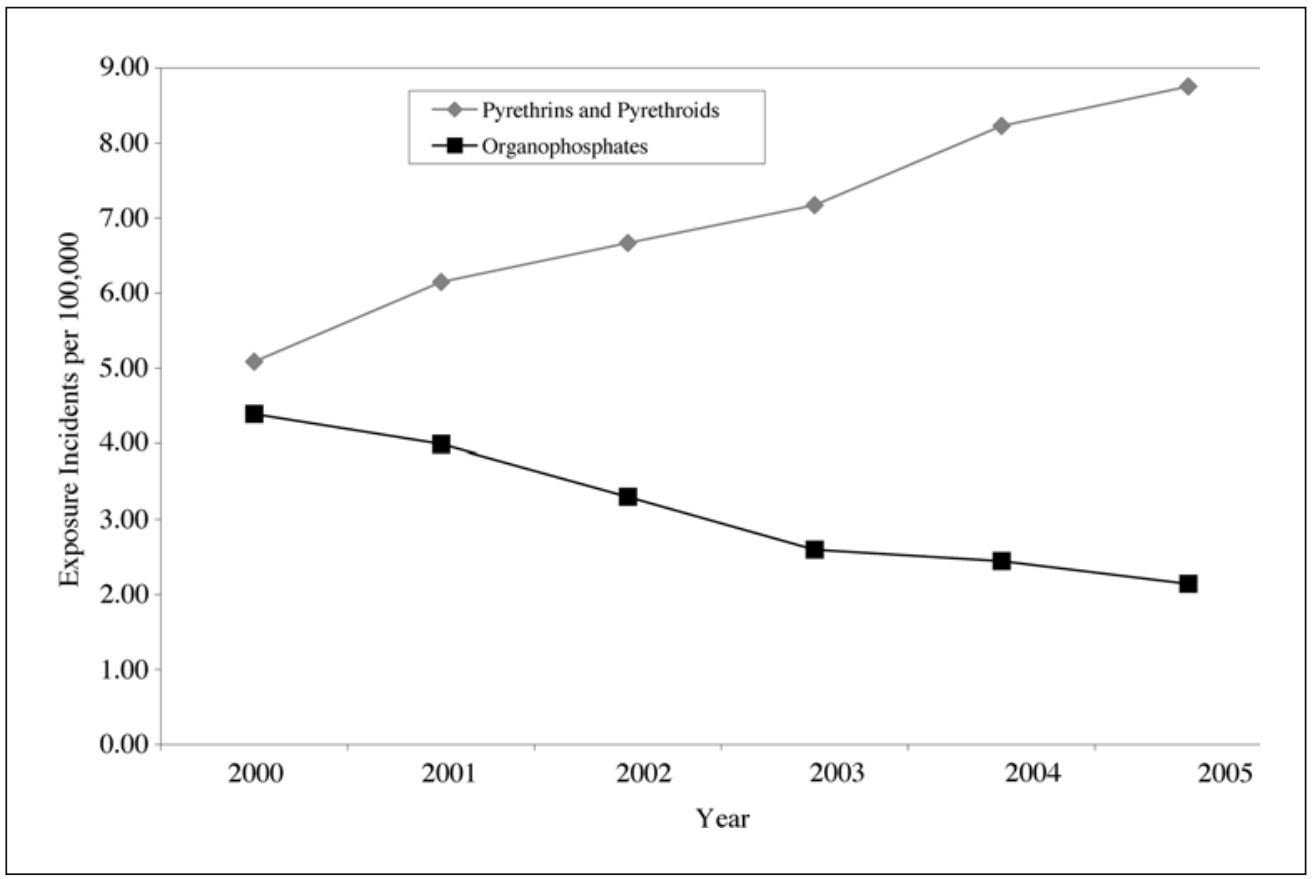

Figure 2: Incidence rate of pyrethrin and pyrethroid, and organophosphate, exposure incidents in the United States, 2000-2005. Organophosphate data taken from [5, 17].

Table 2: Medical outcomes for pyrethrin and pyrethroid exposures in the United States (Toxic Exposure Surveillance System Data, 2000-2005)

\begin{tabular}{|c|c|c|c|c|c|c|}
\hline \multirow[b]{2}{*}{ Year } & \multirow{2}{*}{$\begin{array}{l}\text { \% of Incidents } \\
\text { With Medical } \\
\text { Outcomes } \\
\text { Recorded }\end{array}$} & \multicolumn{5}{|c|}{ Medical Outcome(*) } \\
\hline & & None & Minor & Moderate & Major & Death \\
\hline 2000 & 42.5 & $2,230(38)$ & $2,803(48)$ & $792(14)$ & $23(<1)$ & $0(<1)$ \\
\hline 2001 & 44.3 & $3,032(40)$ & $3,880(51)$ & $725(9)$ & $28(<1)$ & $1(<1)$ \\
\hline 2002 & 44.6 & $3,259(38)$ & $4,551(52)$ & $824(10)$ & $38(<1)$ & $0(<1)$ \\
\hline 2003 & 43.7 & $3,377(37)$ & $4,906(53)$ & $926(10)$ & $25(<1)$ & $3(<1)$ \\
\hline 2004 & 43.9 & $3,899(37)$ & $5,670(53)$ & $1,009(10)$ & $37(<1)$ & $1(<1)$ \\
\hline 2005 & 43.2 & $3,893(35)$ & $6,168(55)$ & $1,090(10)$ & $49(<1)$ & $4(<1)$ \\
\hline \multicolumn{7}{|c|}{ *percentage of medical outcomes } \\
\hline
\end{tabular}

$44 \%$ of medical outcomes were recorded, and the majority of outcomes resulted in either no symptoms (37\%) or minor symptoms (53\%). During the study period, no apparent trend was observed in the number of incidents resulting in major outcomes and death.

\section{DISCUSSION}

TESS data collected from PCCs in the United States showed a clear and consistent increase in cases and incidence rates involving pyrethrins and pyrethroids from 2000 to 2005. The increase was temporally associated with the phase out of organophosphate insecticides from residential uses. This trend was present in all age groups, exposure reasons, and in the number of incidents treated in a health care facility.

Regulatory restriction of access to pesticides may have a complicated effect on the incidence of human exposures, and the results of this study are consistent with other investigations. As an example, an investigation in Sri Lanka examined pesticide exposures after the regulatory restriction and banning of some of the 
more hazardous organophosphate insecticides [22]. Although the study concluded that there was a decreased proportion of case fatalities associated with organophosphates as a result of that regulatory decision, mortality incidents due to an alternative to organophosphates, endosulfan, increased. It is difficult to obtain timely and accurate statistics on pesticide sales and usage, and this factor limits the ability to assess whether the phase-out of organophosphates from residential applications has been associated with increased sales and usage of pyrethrin and pyrethroid insecticides in the United States. In addition to the impact of the risk mitigation decision for organophosphates, there is another factor that potentially influenced the increasing number of incidents involving pyrethrin and pyrethroids. The emergence of West Nile Virus coincided with the study period for this investigation, and several pyrethroid insecticides are registered for public health uses for mosquito control in the United States. While these public health uses could have a significant impact on calls received by PCCs, the manner in which data are collected and reported in TESS do not allow further analysis of their overall impact.

The majority of pyrethrin and pyrethroid exposures in this study resulted in minor or no symptoms. The fact that the majority of incidents were accidental and not intentional is a possible explanation for the low severity of clinical outcomes. The total number of pyrethrin and pyrethroid cases with major medical outcomes and mortality were very low and consistent with the low toxicity of pyrethrins and pyrethroids to humans. These findings suggest that the increase in incidents has not resulted in a significant public health impact in terms of major morbidity and mortality.

Given the relatively low percentage of recorded cases with medical outcomes, we should note that there are limitations in deriving conclusions about medical outcomes from these incidents. If the medical outcome had been reported more frequently, we would have a better understanding of the impact of the phase out on the severity of medical outcomes. There are also other limitations to TESS data, such as underreporting of poisoning incidents [5,23].

The proportion of exposures treated in a health care facility remained constant throughout the study period, while the number of exposures treated in a health care facility increased. This finding is relevant to medical providers and PCCs because they are likely to receive more patients seeking advice and evaluation for pyrethroid and pyrethrin exposure. The clinical features of pyrethroid exposure have been thoroughly reviewed in recent literature [9]. Dermal and inhalation exposure can result in transient paraesthesias and upper airway irritation, while ingestion can result in systemic intoxication that includes nausea, vomiting, abdominal pain, increased secretions, and in severe cases, coma and seizures.

This investigation noted a significant increase in cases involving pyrethrins and pyrethroids associated with the US EPA's regulatory decision to phase out organophosphate insecticides in residential environments. Our findings are consistent with the finding of other investigations. The restricting or banning of access to certain pesticides may have a significant effect on exposures to alternative classes of pesticides. Medical toxicologists and PCC personnel should be prepared to respond to an increasing number of exposures involving pyrethrin and pyrethroid insecticides.

The authors have no potential financial conflicts of interest to report.

\section{REFERENCES}

1. U.S. Environmental Protection Agency (EPA) [webpage on the Internet]. Interim Reregistration Eligibility Decision for Chlorpyrifos. EPA 738-R-01-007. 2002 Feb. Available from: http://www.epa.gov/oppsrrd1/REDs/chlorpyrifos_ired.pdf

2. U.S. Environmental Protection Agency (EPA) [webpage on the Internet]. Interim Reregistration Eligibility Decision: Diazinon. EPA 738-R-04-006. 2004 May. Available from: http://www.epa.gov/oppsrrd1/REDs/diazinon_ired.pdf

3. U.S. Environmental Protection Agency (EPA) [webpage on the Internet]. Chlorpyrifos Revised Risk Assessment and Agreement with Registrants. 2000 Jun. Available from: http://www.epa.gov/pesticides/op/chlorpyrifos/agreement.pdf

4. U.S. Environmental Protection Agency (EPA) [webpage on the Internet]. Diazinon Revised Risk Assessment and Agreement with Registrants. 2001 Jan. Available from:

http://www.epa.gov/pesticides/op/diazinon/agreement.pdf

5. Sudakin DL, Power LE. Organophosphate Exposures in the United States: A Longitudinal Analysis of Incidents Reported to Poison Centers. J Toxicol Environ Health A 2007;70:141-147.

6. Konradsen F, van der Hoek W, Cole DC, Hutchinson G, Daisley H, Singh S, et al. Reducing acute poisoning in developing countries-options for restricting the availability of pesticides. Toxicology 2003;192:249-261.

7. Gunnell D, Eddleston M. Suicide by intentional ingestion of pesticides: a continuing tragedy in developing countries. Int $J$ Epidemiol 2003;32:902-909.

8. Sudakin DL. Pyrethroid insecticides: advances and challenges in biomonitoring. Clin Toxicol 2006;44:31-37.

9. Bradberry SM, Cage SA, Proudfoot AT, Vale JA. Poisoning due to pyrethroids. Toxicol Rev. 2005;24:93-106.

10. Soderlund DM, Clark JM, Sheets LP, Mullin LS, Piccirillo VJ, Sargent D, et al. Mechanisms of pyrethroid neurotoxicity: implications for cumulative risk assessment. Toxicology.

2002; 171:3-59.

11. Proudfoot AT. Poisoning due to pyrethrins. Toxicol Rev. 2005;24:107-113.

12. Litovitz TL, Klein-Schwartz W, White S, Cobaugh DJ, Youniss J, Omslaer JC, et al. 2000 annual report of the American Association of Poison Control Centers Toxic Exposure Surveillance System. Am J Emerg Med.

2001; 19:337-395.

13. Litovitz TL, Klein-Schwartz W, Rodgers Jr. GC, Cobaugh DJ, Youniss J, Omslaer JC, et al. 2001 annual report of the American Association of Poison Control Centers Toxic Exposure Surveillance System. Am J Emerg Med. 2002;20:391-452. 
14. Watson WA, Litovitz TL, Rodgers Jr. GC, Klein-Schwartz W, Youniss J, Rose SR, et al. 2002 Annual Report of the American Association of Poison Control Centers Toxic Exposure Surveillance System. Am J Emerg Med. 2003;21:353-421.

15. Watson WA, Litovitz TL, Klein-Schwartz W, Rodgers Jr. GC, Youniss J, Reid N, et al. 2003 Annual Report of the American Association of Poison Control Centers Toxic Exposure Surveillance System. Am J Emerg Med. 2004;22:335-404.

16. Watson WA, Litovitz TL, Rodgers Jr. GC, Klein-Schwartz W, Reid N, Youniss J, et al. 2004 Annual Report of the American Association of Poison Control Centers Toxic Exposure Surveillance System. Am J Emerg Med. 2005;23:589-666.

17. Lai MW, Klein-Schwartz W, Rodgers Jr. GC, Abrams JY, Haber DA, et al. 2005 Annual Report of the American Association of Poison Control Centers' National Poisoning and Exposure Database. Clin Tox. 2006; 44:803-932.

18. Watson WA, Litovitz TL, Belson MG, Funk Wolkin AB, Patel M, Schier JG, et al. The Toxic Exposure Surveillance System (TESS): Risk assessment and real-time toxicovigilance across United States poison centers. Toxicol Appl Pharmacol. 2005;207:604-610.
19. Litovitz TL, Klein-Schwartz W, White S, Cobaugh DJ, Youniss J, Drab A, et al. 1999 annual report of the American Association of Poison Control Centers Toxic Exposure Surveillance System. Am J Emerg Med. 2000;18:517-574.

20. Litovitz TL, Felberg L, White S, Klein-Schwartz W. 1995 annual report of the American Association of Poison Control Centers Toxic Exposure Surveillance System. Am J Emerg Med. 1996; $14: 487-537$.

21. American Association of Poison Control Centers (AAPCC) [webpage on the Internet]. Instructions for the American Association of Poison Control Centers Toxic Exposure Surveillance System (TESS), Effective November 1, 2001, Required Definitions and Fields for TESS 2002. 2001. Available from: http://www.aapcc.org

22. Roberts DM, Karunarathna A, Buckley NA, Manuweera G, Sheriff $\mathrm{MH}$, Eddleston $\mathrm{M}$. Influence of pesticide regulation on acute poisoning deaths in Sri Lanka. Bull World Health Organ. 2003;81:789-798.

23. Hamilton RJ, Goldfrank LR. Poison center data and the Pollyanna phenomenon. J Toxicol Clin Toxicol. 1997;35:21-23. 